\title{
Strange VLF bursts in northern Scandinavia: case study of the afternoon "mushroom-like" hiss on 8 December 2013
}

\author{
J. Manninen ${ }^{1}$, N. G. Kleimenova ${ }^{2,3}$, A. Kozlovsky ${ }^{1}$, I. A. Kornilov ${ }^{4}$, L. I. Gromova ${ }^{5}$, Y. V. Fedorenko ${ }^{4}$, and T. Turunen ${ }^{1}$ \\ ${ }^{1}$ Sodankylä Geophysical Observatory, 99600 Sodankylä, Finland \\ ${ }^{2}$ Schmidt Institute of Physics of the Earth, RAS, Moscow, 123955, Russia \\ ${ }^{3}$ Space Research Institute, RAS, Moscow, 117997, Russia \\ ${ }^{4}$ Polar Geophysical Institute, RAS, Apatity, 184209, Russia \\ ${ }^{5}$ Pushkov Institute of Terrestrial Magnetism, Ionosphere and Radio Wave Propagation, RAS, Troitsk, 142190, Russia
}

Correspondence to: J. Manninen (jyrki.manninen@sgo.fi)

Received: 25 May 2015 - Revised: 7 July 2015 - Accepted: 23 July 2015 - Published: 12 August 2015

\begin{abstract}
We investigate a non-typical very low frequency (VLF) $1-4 \mathrm{kHz}$ hiss representing a sequence of separated noise bursts with a strange "mushroom-like" shape in the frequency-time domain, each one lasting several minutes. These strange afternoon VLF emissions were recorded at Kannuslehto (KAN, $\varphi=67.74^{\circ} \mathrm{N}, \lambda=26.27^{\circ} \mathrm{E} ; L \sim 5.5$ ) in northern Finland during the late recovery phase of the small magnetic storm on 8 December 2013. The left-hand (LH) polarized 2-3 kHz "mushroom caps" were clearly separated from the right-hand $(\mathrm{RH})$ polarized "mushroom stems" at the frequency of about $1.8-1.9 \mathrm{kHz}$, which could match the lower ionosphere waveguide cutoff (the first transverse resonance of the Earth-ionosphere cavity). We hypothesize that this VLF burst sequence could be a result of the modulation of the VLF hiss electron-cyclotron instability from the strong Pc5 geomagnetic pulsations observed simultaneously at ground-based stations as well as in the inner magnetosphere by the Time History of Events and Macroscale Interactions during Substorms mission probe (THEMIS-E; ThE). This assumption is confirmed by a similar modulation of the intensity of the energetic (1-10 keV) electrons simultaneously observed by the same ThE spacecraft. In addition, the data of the European Incoherent Scatter Scientific Association (EISCAT) radar at Troms $\varnothing$ show a similar quasi-periodicity in the ratio of the Hall-to-Pedersen conductance, which may be used as a proxy for the energetic particle precipitation enhancement. Our findings suggest that this strange mushroom-like shape of the considered VLF hiss could be a combined mutual effect of the magnetospheric
\end{abstract}

ULF-VLF (ultra low frequency-very low frequency) wave interaction and the ionosphere waveguide propagation.

Keywords. Magnetospheric physics (solar windmagnetosphere interactions) - radio science (magnetospheric physics) - space plasma physics (wave-particle interactions)

\section{Introduction}

It has been recognised for a long time that ground observations of natural very low frequency (VLF) emissions provide valuable information for understanding ionospheric and magnetospheric processes. These waves are important because of their ability to efficiently interact with energetic electrons in the magnetosphere via cyclotron resonance (e.g. Hobara et al., 2000; Trakhtengerts and Rycroft, 2008), to change the pitch angle of the energetic particles and to precipitate these particles into the ionosphere.

Due to limited number of the VLF ground stations and satellite data, which could cover the source region of the emissions observed on the ground, there are not enough experimental facts to develop an adequate theory of different types of VLF wave generation. Thus, the ground-based VLF observations carried out by the Sodankylä Geophysical Observatory in northern Finland provide some additional data for such investigations. These were recorded by a very high-sensitivity VLF receiver, with the threshold of the order of $10^{-6} \mathrm{nT}$, and two orthogonal magnetic loop antennas oriented to the north-south and east-west, allowing for the 

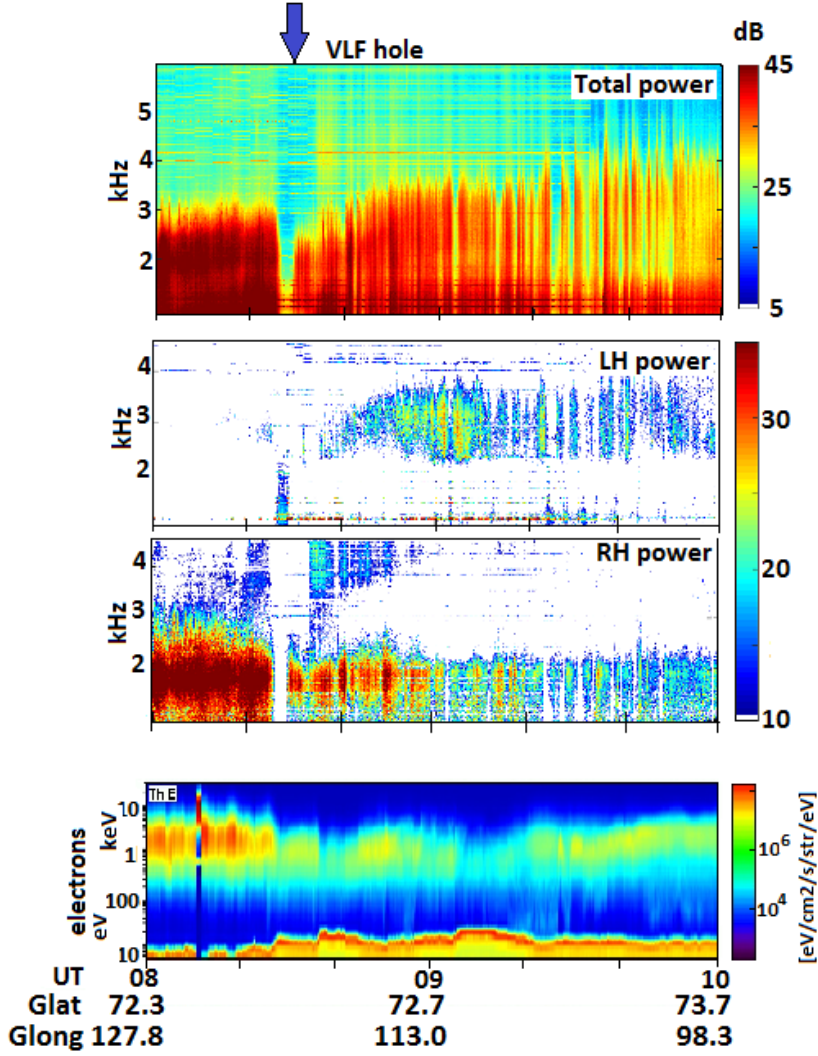

Figure 1. The VLF spectrograms (total, left-handed and righthanded powers) and THEMIS-E electron data from 08:00 to 10:00 UT on 8 December 2013.

polarization of the received signals to be determined. First, it was found that the most interesting VLF events are observed in the wintertime, probably due to better wave scattering from the summer ionosphere at the conjugate Southern Hemisphere. Some non-typical quasi-periodic VLF events obtained during the previous VLF campaigns in 2011-2012 were published in the papers (e.g. Manninen et al., 2012, 2013, 2014a, b).

Here we present new results obtained during the winter VLF campaign (December 2013-January 2014) at Kannuslehto (KAN, with $\varphi=67.74^{\circ} \mathrm{N}, \lambda=26.27^{\circ} \mathrm{E} ; L \sim 5.5$ ), located about $40 \mathrm{~km}$ north-west of the Sodankylä Geophysical Observatory. A detailed description of the applied receiver and data analysis is given by Manninen (2005). We discuss some features of the non-ordinary afternoon VLF hiss, which demonstrated a mushroom structure and was accompanied by simultaneous Pc5 geomagnetic pulsations and fluctuating energetic electrons patches in the inner magnetosphere recorded by the Time History of Events and Macroscale Interactions during Substorms mission probe (THEMIS-E; ThE).
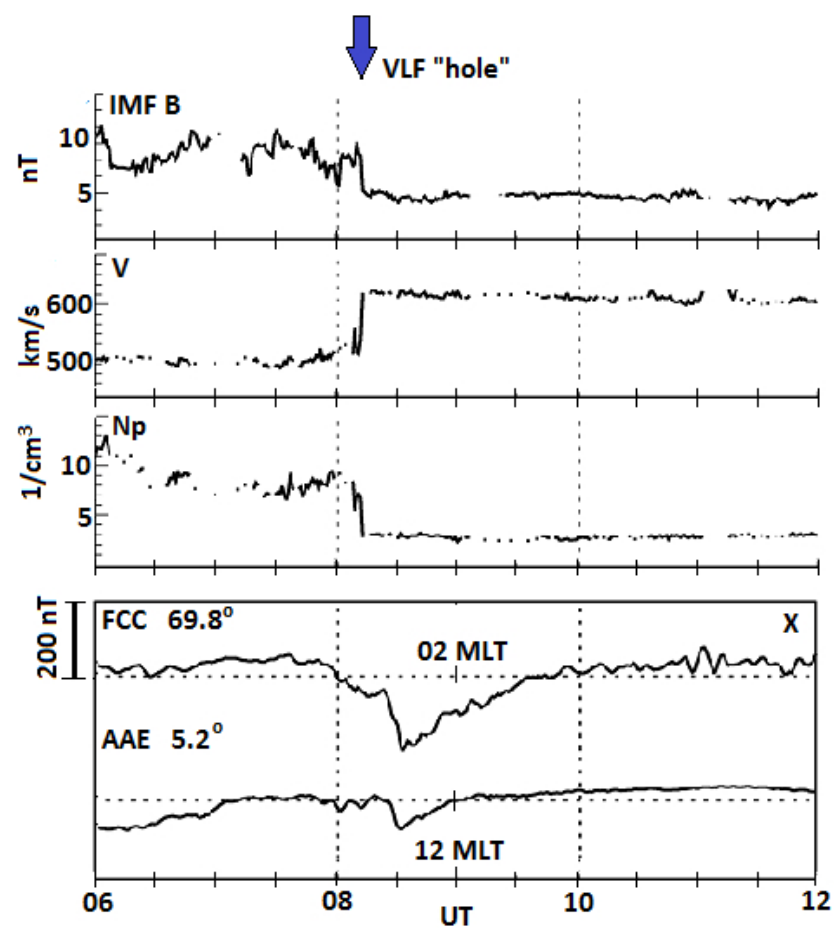

Figure 2. The interplanetary magnetic field (IMF) $B$, solar wind velocity $(V)$ and density $(N p)$ and the night-side substorm at highlatitude station Fort Churchill (FCC) accompanied by negative bay near the equatorial station Addis Ababa (AAE) at noon on 8 December 2013.

\section{Observation results and its discussion}

The considered VLF event was observed on 8 December 2013 during the late recovery phase of a small magnetic storm $\left(\mathrm{Dst}_{\min } \sim-70 \mathrm{nT}\right)$. There were no significant geomagnetic disturbances during the discussed time. Strong, but quite steady hiss at frequencies less than $3 \mathrm{kHz}$ was recorded at KAN from $\sim$ 04:00 UT until $\sim$ 08:25 UT (local magnetic noon at $\sim$ 09:00 UT) when the sharp "hole" (of $3 \mathrm{~min} \mathrm{du}$ ration) in VLF emissions as observed in their spectrograms suddenly occurred (Fig. 1). Before the hole, the waves were right-hand polarised, thus, the station was located in the vicinity of the ionospheric exit area of the wave. Several minutes after the hole, the gradually increasing left-hand polarized waves occurred, indicating that the wave exit area (Yearby and Smith, 1994; Fedorenko et al., 2014) moved away from the station. The unstructured hiss was replaced by some structured VLF spots with a quasi-period of about 3-4 min (Fig. 1).

During the hole, ThE was located in the afternoon sector (the geographic coordinates of the magnetic conjugate point of ThE on the ground, calculated according the model T96, were $\varphi=72.6^{\circ} \mathrm{N}, \lambda=119.2^{\circ} \mathrm{E}, \sim 16: 30 \mathrm{LT}$ ) of the inner magnetosphere (at $X \sim 4 \operatorname{Re}, Y \sim 7.5 \operatorname{Re}, Z \sim-1.1 \operatorname{Re}$ ) and recorded a sharp drop of $1-10 \mathrm{keV}$ electron fluxes (bot- 


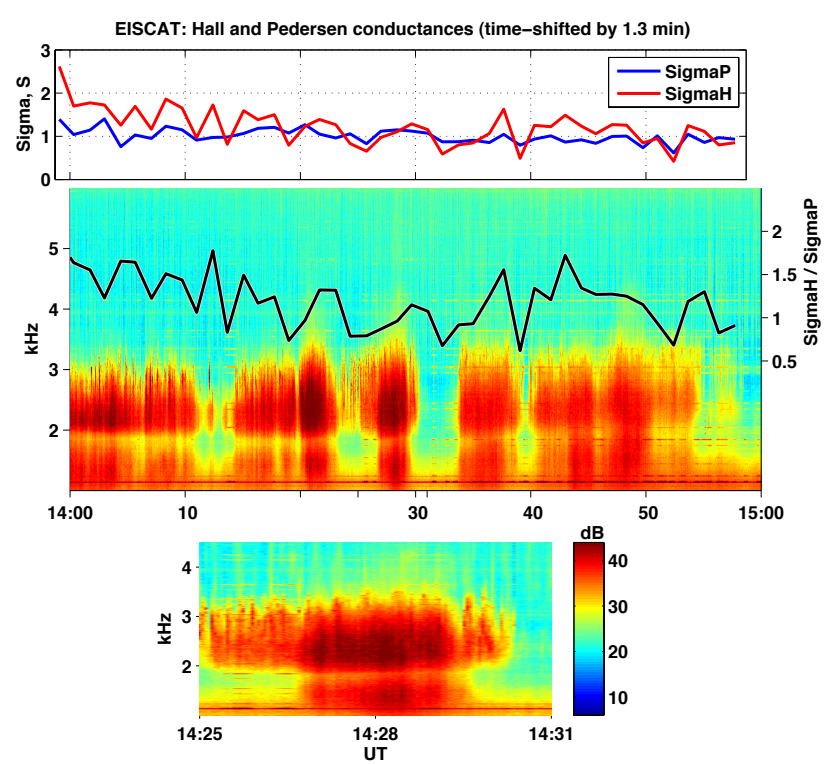

Figure 3. (a) - EISCAT data: the Hall and Pedersen conductances; (b) Total power VLF spectrogram at KAN and the Hall-to-Pedersen conductance ratio (solid black line); (c) - the total power of one individual VLF mushroom.

tom panel in Fig. 1). We also found that the hole followed the drop of the interplanetary magnetic field (IMF) $B$ and solar wind proton density $(\mathrm{Np})$ with the simultaneous jump of the solar wind velocity (Fig. 2). A few minutes later, the nightside high-latitude stations (e.g. FCC, Fig. 2, bottom panel) recorded a small substorm, which was also seen near noon at the equatorial latitudes (e.g. AAE). We hypothesize that the hole in the VLF hiss is a result of the abrupt cessation of the hiss generation due to sharp change of the solar wind and conditions in IMF.

In the afternoon, the VLF hiss structure became more expressive with the shape somewhat similar to mushrooms (Fig. 3b) with averaged durations of about $3 \mathrm{~min}$. It is seen that the "mushroom caps" were clearly separated from their "stems" at the frequency of about $1.8 \mathrm{kHz}$, which corresponds to the Earth-ionosphere waveguide cut-off (the first transverse resonance of the Earth-ionosphere cavity), as reported in many papers (e.g. Yamashita, 1978; Smirnov and Ostapenko, 1986; Ostapenko et al., 2010). On the top of the mushroom caps, one can see a series of quasi-periodic discrete signals (neither chorus nor whistlers). One separated mushroom body is shown in Fig. 3c. The mushroom cap looks like the superposition of bursts of $2-3 \mathrm{kHz}$ discrete signals, modulating at $\sim 20-30 \mathrm{sec}$ and lasting longer than the mushroom stem, and a stronger hiss spot in the centre, coinciding with the mushroom stem hiss. The existence of such a strange spectral structure has not, to our knowledge, been published earlier.

To study the temporal dynamics of the waves, we present here the 3-hour (12:00-15:00 UT) VLF spectrogram (Fig. 4).
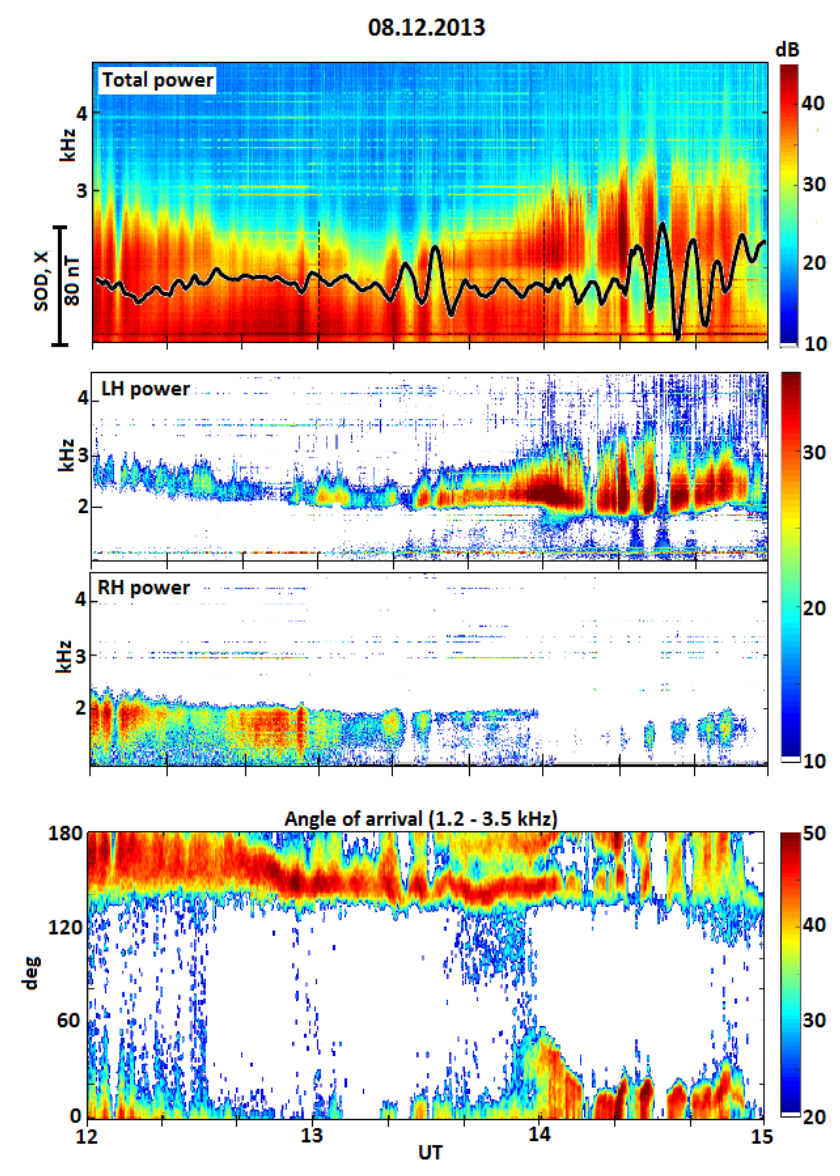

Figure 4. Three upper panels are showing VLF spectrograms (total, left-handed and right-handed powers) and the bottom panel shows angles of arrival of $1.2-3.5 \mathrm{kHz}$ emissions for the 3-hour interval, black curve - Pc5 pulsations from SOD station (in the uppermost panel), from 12:00 to 15:00 UT on 8 December 2013.

It is seen that right-hand (RH) polarized waves, dominating in the beginning of this interval, were gradually replaced by left-hand (LH)ones, which could be interpreted as showing that the distance between our station and the wave source (ionosphere exit area) was increasing. The angles of arrival of the $1.2-3.5 \mathrm{kHz}$ wave (bottom panel in Fig. 4) showed the direction of propagation of the wave to be meridional. $\mathrm{Si}$ multaneous VLF recordings at the Russian station Lovozero, located $\sim 400 \mathrm{~km}$ eastward and equipped additionally with a vertical electric dipole antenna, showed that the studied VLF hiss arrived predominantly from the south (from lower Lshells). It must be underlined that the rather big angle scattering can include some systematic errors in calculations as was shown, e.g. by Strangeways and Rycroft (1980).

According to the estimated direction of the wave arrival from the south, we hypothesize that the studied hiss event, apparently, was excited in the vicinity of (or outside) the plasmapause, which could be located at L-shells much lower than 5 because on the previous day the $\mathrm{Kp}$ value reached $\sim 6$. 
(a)

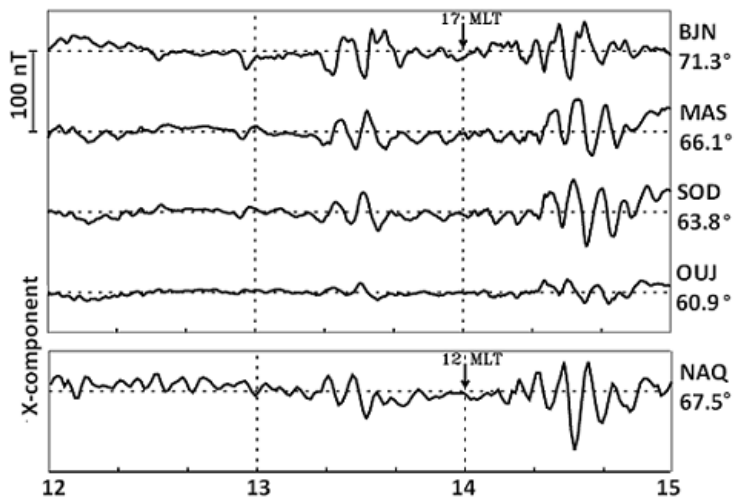

Th E, electrons

(b)
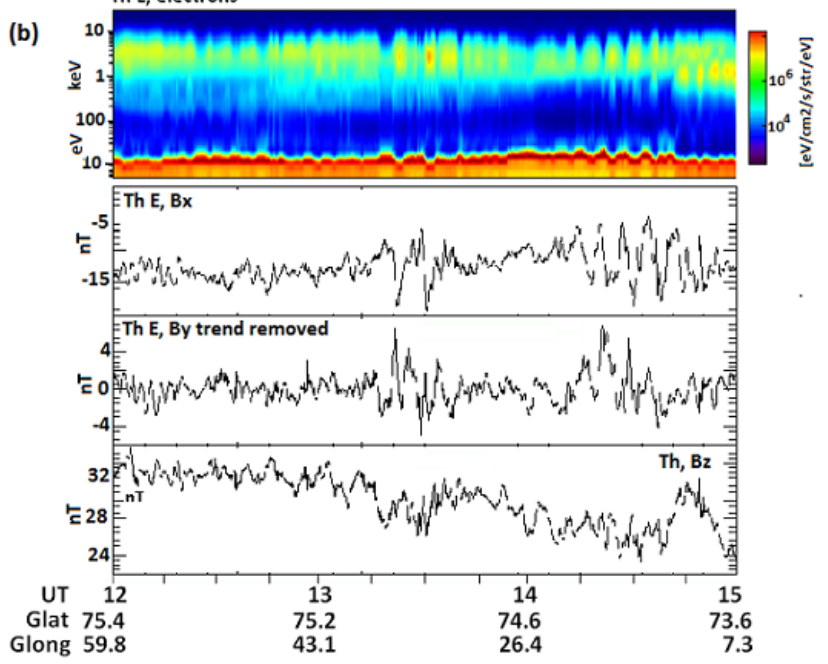

Figure 5. (a) The Pc5 geomagnetic pulsations from four IMAGE stations (BJN, MAS, SOD, and OUJ) and from one Greenland station NAQ. (b) The THEMIS-E data (electron and magnetic field measurements) from 12:00 to 15:00 UT on 8 December 2013.

We found that the considered mushroom-like appearance is controlled by simultaneous Pc5 geomagnetic pulsations (black curve in upper plot in Fig. 4). These pulsations were observed in the afternoon by the IMAGE magnetometer chain (Fig. 5, upper panel). Moreover, they were also observed near noon at Greenland meridional stations (e.g. NAQ, upper panel in Fig. 5). The modulation of VLF hiss due to geomagnetic pulsations was discussed in some papers in the past, including recently (e.g. Sato and Kokubun, 1981; Li et al., 2011).

ThE, which was located at 13:00-15:00 UT in the evening sector (the geographic coordinates of the magnetic conjugate point of ThE on the ground, calculated according the model T96, were $\varphi \sim 74-75^{\circ} \mathrm{N}, \lambda \sim 20-40^{\circ} \mathrm{E}, \sim 17: 00-$ 18:00LT) of the magnetosphere $(X \sim 1.7 \operatorname{Re}, Y \sim 11 R e$, $Z \sim-1.7 R e$ ), also recorded these pulsations mostly in the $\mathrm{B}_{x}$ component (bottom panels in Fig. 5). Rather good agreement is seen between the ground-based and ThE $\mathrm{B}_{x}$ data. Based on some papers (e.g. Denton et al., 2003), we hypoth- esize that the observed Pc5 pulsations mainly represent the poloidal mode of ultra low frequency (ULF) waves with a relatively strong compressional component. Such waves may modulate the growth rate of the electron cyclotron instability (e.g. Trakhtengerts and Rycroft, 2008) and change the pitch angle of the energetic electrons. Actually, for this time, a similar periodicity in the $1-10 \mathrm{keV}$ electron flux intensity can seen in the ThE data (Fig. 5), demonstrating remarkable enhancements of the energetic electron density in the magnetosphere during Pc5 pulsations as was found in some past studies (e.g. Kremser et al., 1981; Nose et al., 1998).

The ionospheric data, derived from the European Incoherent Scatter Scientific Association (EISCAT) radar observations in Troms $\varnothing(\sim 200 \mathrm{~km}$ north-west of KAN $)$ at 14:00-15:00 UT show the Hall and Pedersen conductances (red and blue curves in Fig. 3a). Their ratio (black curve in Fig. 3b) showed quasi-periodic variations, which could be used as a proxy for the enhancements of the energetic charged particle penetration. The ionospheric conductance data in Fig. 3a and b are time-shifted by $1.3 \mathrm{~min}$ (one sampled unit) for better matching with the mushroom-like appearance. Such a phase difference is well explained by the time lag due to the different speeds of the VLF waves and the resonant energetic electrons propagating from their interaction region in the equatorial plane of the magnetosphere (Safargaleev et al., 2003, 2010).

\section{Summary}

The strange shape of $1-4 \mathrm{kHz}$ hiss emissions was found during the late recovery phase of a small magnetic storm. We called this event the "mushroom phenomenon". The comprehensive analysis has been done on the basis of the groundbased VLF and magnetic observations in northern Scandinavia, magnetospheric measurements by the THEMIS-E probe, EISCAT radar data and solar wind and IMF parameters. We found that during this event, a strong wave-particle interaction modulated by strong poloidal Pc5 pulsations took place in the inner magnetosphere.

Our findings suggest that the strange mushroom-like shape of the studied VLF hiss could be a combined mutual effect of the magnetospheric ULF-VLF wave interaction and Earthionosphere waveguide propagation.

Acknowledgements. We are indebted to the director and staff of EISCAT for operating the facility and supplying the data. EISCAT is an international association supported by research organizations in China (CRIRP), Finland (SA), France (CNRS, until the end of 2006), Germany (DFG), Japan (NIPR and STEL), Norway (NFR), Sweden (VR), and the UK (STFC). This work is supported by Program RAS no. 9 and partly by the Russian Foundation for Basic Research (project no. 13-05-00233). N. G. Kleimenova and L. I. Gromova acknowledge the Sodankylä Geophysical Observatory and the Academy of Finland (project 287988) for support during their stay in Sodankylä. 
The topical editor $\mathrm{C}$. Owen thanks one anonymous referee for help in evaluating this paper.

\section{References}

Denton, R. E., Lessard, M. R., and Kistler, L. M.: Radial localization of magnetospheric guided poloidal Pc 4-5 waves, J. Geophys. Res., 108, 1105-1114, doi:10.1029/2002JA009679, 2003.

Fedorenko, Y., Tereshchenko, E., Pilgaev, S., Grigoryev, V., and Blagoveshchenskaya, N.: Polarization of ELF waves generated during "beat-wave" heating experiment near cutoff frequency of the Earth-ionosphere waveguide, Radio Sci., 49, 1254-1264, doi:10.1002/2013RS005336, 2014.

Hobara, Y., Trakhtengerts, V. Y., Demekhov, A. G., and Hayakawa, M.: Formation of electron beams by the interaction of a whistler wave packet with radiation belt electrons, J. Atmos. Sol.-Terr. Phy., 62, 541-552, 2000.

Kremser, G., Korth, A., Fejer, J. A., and Wilken, B.: Observations of quasi-periodic flux variations of energetic ions and electrons associated with Pc 5 geomagnetic pulsations, J. Geophys. Res., 86, 3345-3356. 1981.

Li, W., Thorne, R. M., Bortnik, J., Nishimura, Y., and Angelopoulos, V.: Modulation of whistler mode chorus waves: 1. Role of compressional Pc 4-5 pulsations, J. Geophys. Res., 116, A06205, doi:10.1029/2010JA016312, 2011.

Manninen J.: Some aspects of ELF-VLF emissions in geophysical research, $\mathrm{PhD}$ thesis, Sodankylä Geophysical Observatory Publications, Sodankylä, Finland, 98, 177 pp., 2005.

Manninen, J., Kleimenova, N. G., and Kozyreva, O. V.: New type of ensemble of quasi-periodic, long-lasting VLF emissions at the auroral zone, Ann. Geophys., 30, 1655-1660, doi:10.5194/angeo-30-1655-2012, 2012.

Manninen J., Kleimenova, N. G., Kozyreva, O. V., Bespalov, P. A., and Kozlovsky, A. E.: Non-typical ground-based quasi-periodic VLF emissions observed at $L \sim 5.3$ under quiet geomagnetic conditions at night, J. Atmos. Sol.-Terr. Phy., 99, 123-128. 2013.

Manninen, J., Kleimenova, N. G., Fedorenko, Y. V., Bespalov, P. A., and Turunen, T.: New results of structured VLF emissions observed simultaneously at two closely located stations near $L \sim 5.5$, Ann. Geophys., 32, 1163-1167, doi:10.5194/angeo-321163-2014, 2014a.
Manninen, J., Demekhov, A. G., Titova, E. E., Kozlovsky, A. E., and Pasmanik, D. L.: Quasi-periodic VLF emissions with short-period modulation and their relationship to whistlers: A case study, J. Geophys. Res.-Space, 119, 3544-3557, doi:10.1002/2013JA019743, 2014b.

Nose, M., Iyemori, T., Sugiura, M., Slavin, J. A., Hoffman, R. A., Winningham, J. D., and Sato, N.: Electron precipitation accompanying Pc 5 pulsations observed by the DE satellites and at a ground station, J. Geophys. Res., 103, 17587-17604, 1998.

Ostapenko, A. A., Titova, E. E., Nickolaenko, A. P., Turunen, T., Manninen, J., and Raita, T.: Characteristics of VLF atmospherics near the resonance frequency of the Earth-ionosphere waveguide $1.6-2.3 \mathrm{kHz}$ by observations in the auroral region, Ann. Geophys., 28, 193-202, doi:10.5194/angeo-28-193-2010, 2010.

Safargaleev, V., Kozlovsky, A., Honary, F., Voronin, A., and Turunen, T.: Geomagnetic disturbances on ground associated with particle precipitation during SC, Ann. Geophys., 28, 247-265, doi:10.5194/angeo-28-247-2010, 2010.

Safargaleev, V. V., Pchelkina, E. V., and Vasilyev, A. N.: The time of Alfven wave propagation as inferred from ground based riometer and magnetic data, Geomagn. Aeron., English Translation, 43, 320-325, 2003.

Smirnov, V. S. and Ostapenko, A. A.: Transverse resonances of Earth-ionosphere waveguide in aurora region, Geomagn. Aeron., English Translation, 26, 253-257, 1986.

Sato, N. and Kokubun, S.: Interactions between ELF-VLF emissions and magnetic pulsations and their geomagnetic conjugacy, J. Geophys. Res., 86, 9-18, 1981.

Strangeways, H. J. and Rycroft, M. J.: Systematic errors in VLF direction-finding of whistler ducts-II, J. Atmos. Terr. Phys., 42, 1009-1023, 1980.

Trakhtengerts, V. Y. and Rycroft, M. J.: Whistler and Alfven mode cyclotron masers in space, Cambridge University Press, Cambridge, UK, 2008.

Yamashita, M.: Propagation of tweek atmospherics, J. Atmos. Terr. Phys. 40, 151-156. 1978.

Yearby, K. H. and Smith, A. J.: The polarization of whistlers received on the ground near $L=4$, J. Atmos. Terr. Phys., 56, 1499 1512, 1994. 